\title{
Early Fusion Status after Posterior Lumbar Interbody Fusion with Cortical Bone Trajectory Screw Fixation: A Comparison of Titanium- Coated Polyetheretherketone Cages and Carbon Polyetheretherketone Cages
}

\author{
Hironobu Sakaura ${ }^{1}$, Atsunori Ohnishi ${ }^{2}$, Akira Yamagishi ${ }^{2}$, Tetsuo Ohwada ${ }^{2}$ \\ ${ }^{1}$ Department of Orthopaedic Surgery, Japan Community Healthcare Organization Osaka Hospital, Osaka, Japan \\ ${ }^{2}$ Department of Orthopaedic Surgery, Kansai Rosai Hospital, Amagasaki, Japan
}

\section{Study Design: Retrospective cohort study.}

Purpose: We recently reported that when compared to posterior lumbar interbody fusion (PLIF) using traditional pedicle screw fixation, PLIF with cortical bone trajectory screw fixation (CBT-PLIF) provided favorable clinical outcomes and reduced the incidence of symptomatic adjacent segment pathology, but resulted in relatively lower fusion rates. Since titanium-coated polyetheretherketone (PEEK) cages (TP) could improve and accelerate fusion status after CBT-PLIF, early fusion status was compared between CBT-PLIF using TP and carbon PEEK cages (CP).

Overview of Literature: A systematic review demonstrated that clinical studies at this early stage show similar fusion rates for TP compared to PEEK cages.

Methods: We studied 36 consecutive patients undergoing CBT-PLIF with TP (TP group) and 92 undergoing CBT-PLIF with CP (CP group). On multiplanar reconstruction computed tomography (MPR-CT) at 6 months postoperatively, vertebral endplate cysts (cyst signs) were evaluated and classified as diffuse or local cysts. Early fusion status was assessed by dynamic plain radiographs and MPR-CT at 1 year postoperatively.

Results: The incidences of cyst signs, diffuse cysts, and early fusion rate in the TP and CP groups were $38.9 \%$ and $66.3 \%(p<0.01)$, $16.7 \%$ and $32.6 \%(p=0.07)$, and $83.3 \%$ and $79.3 \%$ ( $p>0.05$ ), respectively. Combining the two groups, 22 of 36 patients with diffuse cysts had nonunion at 1-year follow-up, compared to only three of 92 patients with local cysts or without cyst signs $(p<0.01)$.

Conclusions: Despite having fewer patients with endplate cysts at 6 months (a known risk factor for nonunion), the TP group had the same fusion rate as the CP group at 1-year follow-up. Thus, TP did not accelerate the fusion process after CBT-PLIF.

Keywords: Degenerative lumbar spondylolisthesis; Posterior lumbar interbody fusion; Cortical bone trajectory screw technique; Titanium-coated polyetheretherketone cage; Carbon polyetheretherketone cage

\footnotetext{
Received Jul 9, 2018; Revised Aug 9, 2018; Accepted Aug 15, 2018

Corresponding author: Hironobu Sakaura

Department of Orthopaedic Surgery, Japan Community Healthcare Organization Osaka Hospital, 4-2-78 Fukushima, Fukushima-ku, Osaka city, Osaka 553-0003, Japan

Tel: +81-6-6441-5451, Fax: +81-6-6445-8900, E-mail: sakaura04061023@yahoo.co.jp
} 


\section{Introduction}

Cortical bone trajectory (CBT) screw fixation has been reported to have favorable mechanical properties [1-4]. We recently reported that posterior lumbar interbody fusion (PLIF) with CBT screw fixation (CBT-PLIF) provided better postoperative improvement of clinical symptoms and reduced the incidence of symptomatic adjacent segment disease when compared to PLIF using traditional pedicle screw fixation (PS-PLIF) [5]. However, in our previous study, the fusion rate tended to be lower after CBT-PLIF than after PS-PLIF, although there was no significant difference [5].

Polyetheretherketone (PEEK) has an elastic modulus similar to cortical bone with the addition of carbon fiber [6]. Unfortunately, PEEK is a bioinert material [6]. On the other hand, the benefit of a titanium interface for PEEK for cellular attachment and osteoblastic phenotype expression has been demonstrated by in vitro studies [6-8]. Moreover, titanium-coated PEEK (TP) has osteoconductive activity and increases shear strength in an idealized model [9]. Therefore, since we expected that the TP cage could improve and accelerate fusion status after CBTPLIF, we changed from the carbon fiber-reinforced PEEK cage (CP cage) to the TP cage in January 2016. Thus, early fusion status, including the incidence of vertebral endplate cysts (cyst signs), was compared between CBT-PLIF using $\mathrm{TP}$ and $\mathrm{CP}$ cages.

\section{Materials and Methods}

\section{Patients}

We studied 36 consecutive patients ( 19 men, 17 women; mean age at surgery, 65.3 years; range, $37-83$ years) who underwent single-level CBT-PLIF with TP cages for degenerative lumbar spondylolisthesis from January 2016 (TP group). Fusion areas were L3 to L4 in four patients, L4 to L5 in 28, and L5 to S1 in four. The historical control group consisted of 92 consecutive patients (44 men, 48 women; mean age at surgery, 68.5 years; range, $42-85$ years) who underwent single-level CBT-PLIF with CP cages for degenerative lumbar spondylolisthesis before December 2015 by the same surgeons as for the TP group (CP group). Fusion areas were L1 to L2 in one patient, L2 to L3 in one, L3 to L4 in 15, L4 to L5 in 71, L5 to L6 in one, and L5 to $\mathrm{S} 1$ in three. All patients were considered for surgery because of unresponsiveness to conservative treatment, such as medication and/or epidural block. Age at surgery, sex, and fusion area showed no significant differences between the two groups. The protocol was approved by the Kansai Rosai Hospital ethics committee and written informed consent was obtained from all participants.

\section{Surgical procedure}

In all patients from both groups, CBT-PLIF was performed in the same manner. First, adequate posterior decompression was performed. After intervertebral disc materials were removed, two cages filled with local bone graft were inserted into the bilateral outer sides of the intervertebral space and two or three local bone blocks were inserted medial to the cages. The fused segment was fixed using the CBT screw-rod system (Fig. 1A, B). All patients in both groups wore a thoracolumbosacral orthosis for 3 months postoperatively.

\section{Clinical and radiologic evaluations}

Clinical results were assessed using the Japanese Orthopaedic Association (JOA) scoring system for assessment of low back pain treatment results by the first author (Table 1) [10]. The total JOA score is 29 points in the normal
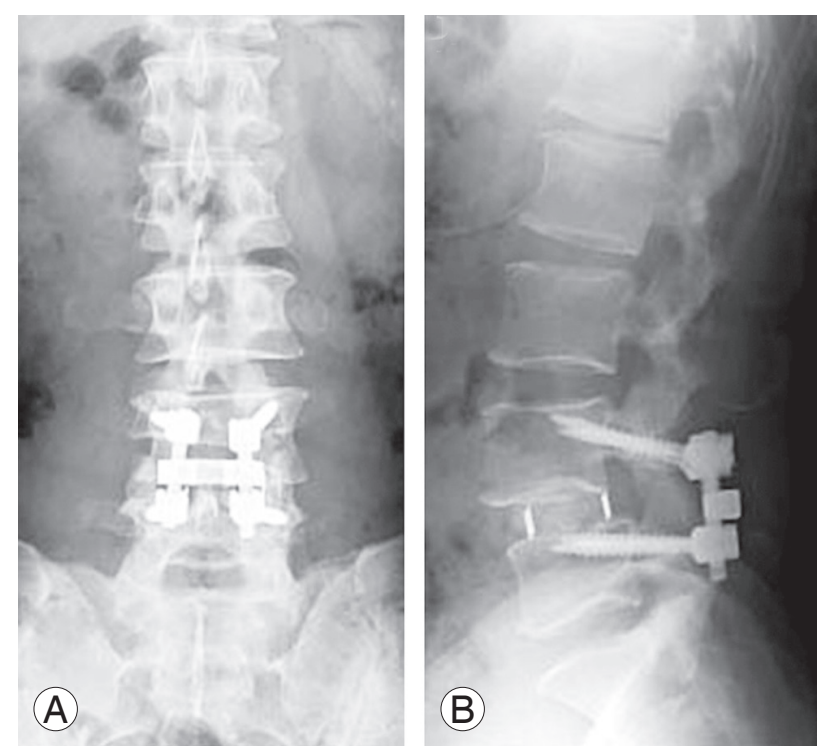

Fig. 1. Radiographs 5 days after posterior lumbar interbody fusion with cortical bone trajectory screw fixation using titanium-coated polyetheretherketone cages. (A) Anteroposterior view. (B) Lateral view in the neutral position. 
Table 1. Japanese Orthopaedic Association scoring system for assessment of the results of treatment for low back pain

\begin{tabular}{|c|c|}
\hline Item & Point range \\
\hline \multicolumn{2}{|l|}{ Subjective symptoms (9 points) } \\
\hline Low-back pain & $3,2,1,0$ \\
\hline Leg pain and/or leg tingling sensation & $3,2,1,0$ \\
\hline Neurogenic intermittent claudication & $3,2,1,0$ \\
\hline \multicolumn{2}{|l|}{ Clinical signs (6 points) } \\
\hline Straight-leg-raising test & $2,1,0$ \\
\hline Leg sensory disorder & $2,1,0$ \\
\hline Loss in leg muscle strength & $2,1,0$ \\
\hline \multicolumn{2}{|c|}{ Restriction of activities of daily living (14 points) } \\
\hline Roll-over & $2,1,0$ \\
\hline Standing up & $2,1,0$ \\
\hline Face-wash & $2,1,0$ \\
\hline Hold leaning forward or standing & $2,1,0$ \\
\hline Prolonged sitting (about $1 \mathrm{hr}$ ) & $2,1,0$ \\
\hline Lifting or holding heavy object & $2,1,0$ \\
\hline Ambulation & $2,1,0$ \\
\hline Bladder function & $0,-3,-6$ \\
\hline Total $^{\text {al }}$ & 29 to -6 \\
\hline
\end{tabular}

${ }^{\text {a) }}$ A normal total Japanese Orthopaedic Association score is 29 points.

population. Postoperative recovery rate of the JOA score was calculated by the following formula: postoperative recovery rate $(\%)=($ postoperative score-preoperative score $) /$ (29-preoperative score) $\times 100$.

At our institute, all patients undergoing PLIF routinely underwent computed tomography (CT) at 6 months postoperatively to examine PS loosening and cage subsidence, and then again at 1 year postoperatively to assess early fusion status. Using multiplanar reconstruction CT (MPR-CT) at 6 months postoperatively, cyst signs were evaluated and classified as diffuse (vertebral endplate cyst larger than one-half of the entire length of an intervertebral cage) (Fig. 2A) or local (a localized, relatively small vertebral endplate cyst) (Fig. 2B) by the first author [11]. Positive cyst signs were defined as circular or oval lowdensity areas around the intervertebral cages on sagittal reconstruction CT images, which had newly developed or became enlarged postoperatively.

Early fusion status was assessed by dynamic plain radiographs and MPR-CT at 1-year postoperatively by the first author. Solid fusion was defined as the condition in which osseous continuity between the vertebrae and grafted
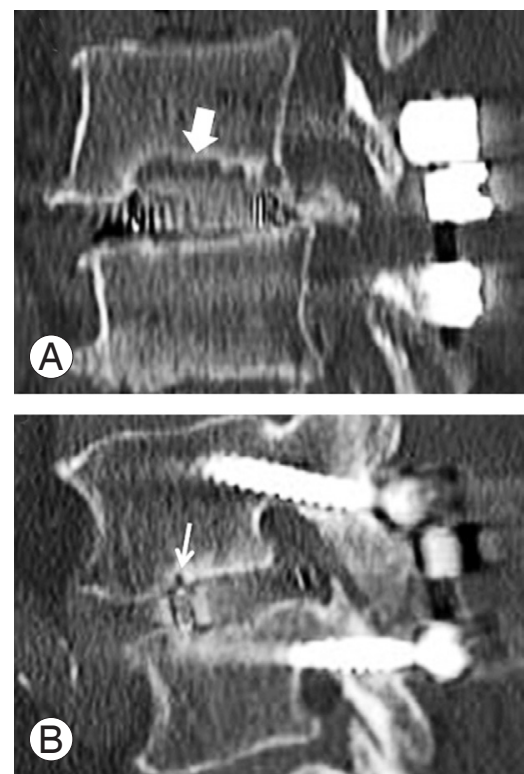

Fig. 2. Sagittal computed tomography images at 6 months postoperatively. (A) A diffuse cyst is defined as a vertebral endplate cyst larger than one-half of an entire length of an intervertebral cage (bold white arrow). (B) A local cyst is defined as a localized, relatively small vertebral endplate cyst (thin white arrow).

bone was achieved on MPR-CT, with neither loosening of the PSs nor motion at the fused segments on lateral flexion-and-extension radiographs. Fusion status was graded as either union in situ (solid fusion without loss of graft height), collapsed union (solid fusion with $\geq 2$-mm cage subsidence into the adjacent vertebral body), or nonunion according to the previously reported criteria [12].

\section{Statistical analysis}

The unpaired $t$-test, Mann-Whitney $U$-test, Wilcoxon signed-rank test, Kruskal-Wallis test, and Fisher's exact probability test were used for statistical analysis with JMP ver. 5.0.1 (SAS Institute Inc., Cary, NC, USA), as appropriate. Values of $p<0.05$ were considered significant.

\section{Results}

\section{Clinical results}

The mean JOA score improved significantly, from 13.0 points preoperatively to 22.8 points, at 1 year postoperatively (mean recovery rate, $62.8 \%$ ) in the TP group, and from 13.9 to 23.7 points (mean recovery rate, $65.6 \%$ ), respectively, in the $\mathrm{CP}$ group (Table 2). There were no sig- 
Table 2. Clinical outcomes in the two groups (TP group and CP group)

\begin{tabular}{lccc} 
Variable & TP group (n=36) & CP group (n=92) & $p$-value \\
JOA score before surgery (points) & $13.0 \pm 4.4$ & $13.9 \pm 4.5$ & 0.496 \\
JOA score at 1-year follow-up (points) & $22.8 \pm 4.9^{\text {a) }}$ & $23.7 \pm 3.6^{\text {a) }}$ & 0.342 \\
\hline Recovery rate of JOA score at 1-year follow-up (\%) & $62.8 \pm 26.7$ & $65.6 \pm 21.6$ & 0.550 \\
\hline
\end{tabular}

Values are presented as mean \pm standard deviation.

JOA, Japanese Orthopaedic Association, TP, titanium-coated PEEK cage; CP, carbon fiber reinforced PEEK cage; PEEK, polyetheretherketone.

${ }^{a}$ Significantly higher than before surgery (Wilcoxon signed-rank test, $p<0.001$ ).

Table 3. Cyst signs in the two groups (TP group and CP group)

\begin{tabular}{lccc} 
Variable & TP group $(\mathrm{n}=36)$ & CP group $(\mathrm{n}=92)$ & $p$-value \\
Total cyst signs & $14(38.9)$ & $61(66.3)$ & 0.00480 \\
Diffuse cyst & $6(16.7)$ & $30(32.6)$ & 0.0724 \\
Local cyst & $8(22.2)$ & $31(33.7)$ & 0.207 \\
\hline
\end{tabular}

Values are presented as number of cases (\%).

TP, titanium-coated PEEK cage; CP, carbon fiber reinforced PEEK cage; PEEK, polyetheretherketone.

nificant differences in preoperative JOA score, JOA score at 1-year follow-up, and JOA score recovery rate between the two groups (Table 2).

\section{Cyst signs}

At 6 months postoperatively, cyst signs were found in 14 of 36 patients (38.9\%; diffuse in six, local in eight) in the TP group, and 61 of 92 (66.3\%; diffuse in 30 and local in 31) in the CP group (Table 3). The total incidence of cyst signs was significantly lower in the TP than in the $\mathrm{CP}$ groups (Table 3). The incidence of diffuse cysts in the TP group decreased to approximately half that of the $\mathrm{CP}$ group, although no significant difference was found between the groups (Table 3).

\section{Fusion status}

At 1-year follow-up, union in situ, collapsed union, and nonunion were achieved in 25 , five, and six of 36 patients, respectively, in the TP group, and 50, 23, and 19 of 92 patients, respectively, in the $\mathrm{CP}$ group. Thus, solid spinal fusion was achieved in 30 of 36 (early fusion rate, 83.3\%) and 79 of 92 (early fusion rate, 79.3\%) patients, respectively (Table 4). The rate of collapsed union tended to be lower in the TP than in the $\mathrm{CP}$ groups, although no significant difference was found (Table 4). Altogether, early fusion status at 1-year follow-up showed no significant
Table 4. Fusion status in the two groups (TP group and CP group)

\begin{tabular}{lccc} 
Variable & TP group $(\mathrm{n}=36)$ & CP group $(\mathrm{n}=92)$ & $p$-value \\
Total union & $30(83.3)$ & $73(79.3)$ & 0.610 \\
Union in situ & $25(69.4)$ & $50(54.3)$ & 0.120 \\
\hline Collapsed union & $5(13.9)$ & $23(25.0)$ & 0.0845 \\
\hline
\end{tabular}

Values are presented as number of cases (\%).

TP, titanium-coated PEEK cage; CP, carbon fiber reinforced PEEK cage; PEEK, polyetheretherketone.

difference between the two groups (Table 4).

Combining both groups, 22 of 36 patients (61.1\%) with diffuse cysts had nonunion at 1 year postoperatively, compared to only two of 39 (5.1\%) with local cysts and only one of $53(1.9 \%)$ without cyst signs $(p<0.01)$.

\section{Discussion}

We recently reported that single-level CBT-PLIF for degenerative lumbar spondylolisthesis provided favorable postoperative improvement of clinical symptoms and reduced the incidence of symptomatic adjacent segment disease compared to single-level PS-PLIF for the same pathologic condition [5]. However, our previous study showed that the fusion rate tended to be lower after CBTPLIF than after PS-PLIF, though there was no significant difference [5]. Although a CBT screw demonstrates greater fixation strength for each individual screw than a traditional PS [1-3,13], biomechanical cadaver and finite element studies revealed that the traditional PS-rods construct is stiffer than the CBT screw-rods construct during axial rotation and lateral bending $[4,13]$. Inferior stiffness of the CBT screw-rods construct to that of the traditional PS-rods construct during axial rotation and lateral bending may result in a relatively lower fusion rate after CBTPLIF than after PS-PLIF. Therefore, we believe that CBTPLIF needs intervertebral cages that provide superior early postoperative fixation strength and have osteoconductive 
activity to improve a successful fusion rate.

Currently, the most popular interbody cages for PLIF are made of titanium alloys or PEEK. Titanium has osseointegration activity [14]. However, a significant disadvantage of titanium is the discrepancy in its elastic modulus compared to bone, which potentially leads to stress shielding and subsidence $[7,14,15]$. On the other hand, PEEK has an elastic modulus similar to cortical bone with the addition of carbon fiber, but its weakness is that it is bioinert [6]. A comparative clinical study showed that the fusion rate after transforaminal lumbar interbody fusion with traditional PS fixation (PS-TLIF) using titanium cages was superior to that following PS-TLIF using PEEK cages [16]. On the other hand, another comparative clinical study showed no significant difference in bone union rate between PS-TLIF using titanium versus PEEK cages [17]. Given these results, this issue remains controversial.

Most recently, TP cages that combine the favorable biomechanical properties of PEEK and the bioactivities of titanium have been available for PLIF. Some in vitro studies demonstrated the benefit of a titanium interface for PEEK for cellular attachment and osteoblastic phenotype expression [6-8]. In addition to these biological advantages, TP has osteoconductive activity and increases shear strength in an idealized model [9]. Since we expected that TP cages could improve the successful early fusion rate after CBT-PLIF based on these advantages, we changed from CP to TP cages in January 2016. Therefore, early fusion status including cyst signs was compared between CBT-PLIF using TP and CP cages. However, in the present comparative clinical study, the fusion rate at 1 year postoperatively was not significantly different between the $\mathrm{TP}$ and CP groups. A systematic review also has shown that clinical studies at this early stage demonstrate similar fusion rates for TP combined compared to PEEK cages [18]. On the other hand, the rate of collapsed union in the $\mathrm{TP}$ group decreased to approximately half that of the $\mathrm{CP}$ group, although there was no significant difference between the groups. Kotsias et al. [19] also reported reduced subsidence in the TP cage compared to the PEEK cage cohorts after anterior cervical fusion. We speculated that TP cages, which provide greater early postoperative fixation strength than CP cages due to the titanium plasmapore surface, could decrease a collapsed union rate by decreasing micromotion on the interface between a vertebral endplate and a cage. These results indicated that the TP cages could potentially reduce subsidence.
Regarding cyst signs, the incidence of cyst signs is reportedly significantly higher after CBT-PLIF than after PS-PLIF [11]. Biomechanical studies showed that the CBT screw-rods construct has inferior stiffness compared to the traditional PS-rods construct during axial rotation and lateral bending $[4,13]$. This inferior stiffness of the CBT screw-rods construct during axial rotation and lateral bending can produce micromotion, which might result in a higher incidence of cyst signs after CBT-PLIF than after PS-PLIF. Our study showed that a diffuse cyst is a significant predictor of nonunion at 1 year after CBT-PLIF. Similar results in previous reports support this finding $[16,17]$. In this comparative study, the incidence of diffuse cysts in the TP group decreased to approximately half that of the CP group, although no significant difference was found between the groups. Thus, TP cages, which provide greater early postoperative fixation strength than CP cages and have osteoconductive activity, may reduce the incidence of diffuse cysts by approximately half that of the $\mathrm{CP}$ group. On the other hand, the fusion rate at 2 years after PS-TLIF was reportedly increased compared to that at 1 year postoperatively $[16,17]$. Considering these results, although early fusion status at 1-year follow-up showed no significant difference between the groups, we expected that the fusion rate at 2 years postoperatively might increase more in the TP than in the CP groups.

Our study has several limitations. The 1-year follow-up period was relatively short. However, since TP cages could accelerate the fusion process after CBT-PLIF, our purpose was to compare early fusion status at 1 year postoperatively between CBT-PLIF using TP versus carbon PEEK cages. This study was retrospective in nature. The osteoporosis status, which can affect fusion status after PLIF, was not measured. The number of patients was much smaller in the TP than in the CP groups. A further longterm study including osteoporosis status with a larger TP group will be needed.

\section{Conclusions}

In conclusion, clinical functional outcomes at 1 year after CBT-PLIF showed no significant difference between the TP and CP groups. Despite having fewer patients with endplate cysts at 6 months (a known risk factor for nonunion) and cage subsidence, the TP group had the same fusion rate as the CP group at 1 year. Thus, the TP cage did not accelerate the fusion process after CBT-PLIF. 


\section{Conflict of Interest}

No potential conflict of interest relevant to this article was reported.

\section{Author Contributions}

Conception and design: HS, AO, AY, TO; data acquisition: HS; analysis of data: HS; drafting of the manuscript: HS; critical revision: HS, AO, AY, TO; and supervision: TO.

\section{References}

1. Santoni BG, Hynes RA, McGilvray KC, et al. Cortical bone trajectory for lumbar pedicle screws. Spine J 2009;9:366-73.

2. Matsukawa K, Yato Y, Kato T, Imabayashi H, Asazuma T, Nemoto K. In vivo analysis of insertional torque during pedicle screwing using cortical bone trajectory technique. Spine (Phila Pa 1976) 2014;39:E240-5.

3. Baluch DA, Patel AA, Lullo B, et al. Effect of physiological loads on cortical and traditional pedicle screw fixation. Spine (Phila Pa 1976) 2014;39:E1297-302.

4. Perez-Orribo L, Kalb S, Reyes PM, Chang SW, Crawford NR. Biomechanics of lumbar cortical screw-rod fixation versus pedicle screw-rod fixation with and without interbody support. Spine (Phila Pa 1976) 2013;38:635-41.

5. Sakaura H, Miwa T, Yamashita T, Kuroda Y, Ohwada T. Posterior lumbar interbody fusion with cortical bone trajectory screw fixation versus posterior lumbar interbody fusion using traditional pedicle screw fixation for degenerative lumbar spondylolisthesis: a comparative study. J Neurosurg Spine 2016;25:591-5.

6. Kurtz SM, Devine JN. PEEK biomaterials in trauma, orthopedic, and spinal implants. Biomaterials 2007;28:4845-69.

7. Rao PJ, Pelletier MH, Walsh WR, Mobbs RJ. Spine interbody implants: material selection and modification, functionalization and bioactivation of surfaces to improve osseointegration. Orthop Surg 2014;6:81-9.

8. Sagomonyants KB, Jarman-Smith ML, Devine JN, Aronow MS, Gronowicz GA. The in vitro response of human osteoblasts to polyetheretherketone (PEEK) substrates compared to commercially pure titanium. Biomaterials 2008;29:1563-72.
9. Walsh WR, Bertollo N, Christou C, Schaffner D, Mobbs RJ. Plasma-sprayed titanium coating to polyetheretherketone improves the bone-implant interface. Spine J 2015;15:1041-9.

10. Yone K, Sakou T, Kawauchi Y, Yamaguchi M, Yanase M. Indication of fusion for lumbar spinal stenosis in elderly patients and its significance. Spine (Phila Pa 1976) $1996 ; 21: 242-8$.

11. Kaito T, Fujiwara H, Makino T, Kashii M, Sugiura T. Strengths and limitations of pedicle screw fixation with cortical bone trajectory (CBT). Orthop Surg Traumatol 2014;57:1583-9.

12. Yamamoto T, Ohkohchi T, Ohwada T, Kotoku H, Harada N. Clinical and radiological results of PLIF for degenerative spondylolisthesis. J Musculoskelet Res 1998;2:181-95.

13. Matsukawa K, Yato Y, Imabayashi H, Hosogane N, Asazuma T, Nemoto K. Biomechanical evaluation of the fixation strength of lumbar pedicle screws using cortical bone trajectory: a finite element study. J Neurosurg Spine 2015;23:471-8.

14. Fujibayashi S, Takemoto M, Neo M, et al. A novel synthetic material for spinal fusion: a prospective clinical trial of porous bioactive titanium metal for lumbar interbody fusion. Eur Spine J 2011;20:1486-95.

15. Ma R, Tang T. Current strategies to improve the bioactivity of PEEK. Int J Mol Sci 2014;15:5426-45.

16. Nemoto O, Asazuma T, Yato Y, Imabayashi H, Yasuoka $\mathrm{H}$, Fujikawa A. Comparison of fusion rates following transforaminal lumbar interbody fusion using polyetheretherketone cages or titanium cages with transpedicular instrumentation. Eur Spine J 2014;23:2150-5.

17. Tanida S, Fujibayashi S, Otsuki B, et al. Vertebral endplate cyst as a predictor of nonunion after lumbar interbody fusion: comparison of titanium and polyetheretherketone cages. Spine (Phila Pa 1976) 2016;41:E1216-E22.

18. Assem Y, Mobbs RJ, Pelletier MH, Phan K, Walsh WR. Radiological and clinical outcomes of novel Ti/PEEK combined spinal fusion cages: a systematic review and preclinical evaluation. Eur Spine J 2017;26:593-605.

19. Kotsias A, Mularski S, Cabraja M, Kombos T, Suss O. Fusion characteristics in ACDF with partially titanium-coated PEEK cages. Eur Spine J 2013;22:2582669. 